Canad. Math. Bull. Vol. 37 (2), 1994 pp. 154-161

\title{
THE AVERAGE EDGE ORDER OF 3-MANIFOLD COLOURED TRIANGULATIONS
}

\author{
MARIA RITA CASALI
}

ABSTRACT. If $K$ is a triangulation of a closed 3-manifold $M$ with $E_{0}(K)$ edges and $F_{0}(K)$ triangles, then the average edge order of $K$ is defined to be

$$
\mu_{0}(K)=\frac{3 F_{0}(K)}{E_{0}(K)} .
$$

In [8], the relations between this quantity and the topology of $M$ are investigated, especially in the case of $\mu_{0}(K)$ being small (where the study relies on Oda's classification of triangulations of $\mathbb{S}^{2}$ up to eight vertices-see [9]). In the present paper, the attention is fixed upon the average edge order of coloured triangulations; surprisingly enough, the obtained results are perfectly analogous to Luo-Stong' ones, and may be proved with little effort by means of edge-coloured graphs representing manifolds.

1. Introduction and preliminaries. If $K$ is a triangulation of a closed 3-manifold $M$ with $E_{0}(K)$ edges and $F_{0}(K)$ triangles, then the average edge order of $K$ is defined to be

$$
\mu_{0}(K)=\frac{3 F_{0}(K)}{E_{0}(K)} .
$$

In [8], the relations between this quantity and the topology of $M$ are investigated, especially in the case of $\mu_{0}(K)$ being small (where the study relies on Oda's classification of triangulations of the 2 -sphere up to eight vertices).

The main results are collected in the following proclaim (where $\mathbb{S}^{n}$ and $\mathbb{S}^{2} \tilde{x} \mathbb{S}^{1}$ respectively denote the $n$-dimensional sphere and the twisted 2 -sphere bundle over $\mathbb{S}^{1}$ ):

THEOREM I [8]. Let $K$ be any triangulation of a closed connected 3-manifold $M$. Then:

(a) $3 \leq \mu_{0}(K)<6$, equality holds if and only if $K$ is the triangulation of the boundary of a 4-simplex.

(b) For any $\epsilon>0$, there are triangulations $K_{1}$ and $K_{2}$ of $M$ such that $\mu_{0}\left(K_{1}\right)<4.5+\epsilon$ and $\mu_{0}\left(K_{2}\right)>6-\epsilon$.

(c) If $\mu_{0}(K)<4.5$, then $K$ is a triangulation of $\mathbb{S}^{3}$.

(d) If $\mu_{0}(K)=4.5$, then $K$ is a triangulation of $\mathbb{S}^{3}, \mathbb{S}^{2} \times \mathbb{S}^{1}$, or $\mathbb{S}^{2} \tilde{\times} \mathbb{S}^{1}$. Furthermore, in the last two cases, the triangulations can be described.

Work performed under the auspicies of the G.N.S.A.G.A. of the C.N.R. (National Research Council of Italy) and financially supported by M.U.R.S.T. of Italy (project "Geometria Reale e Complessa").

Received by the editors June 18, 1992; revised January 28, 1993 and September 7, 1993.

AMS subject classification: 57Q15, 05C10, 57M15.

(c) Canadian Mathematical Society 1994. 
The present paper works within the polyhedral category (P.L.) - for which we refer to [11], - and fixes the attention upon coloured triangulations of 3-manifolds, wherefrom now on-we write " $n$-manifold" instead of "closed connected $n$-dimensional PLmanifold".

Roughly speaking, a coloured triangulation of an $n$-manifold $M$ is a pseudocomplex $\bar{K}$ (i.e. two simplices may have more than one common face) such that $|\bar{K}| \cong M$, and with the vertices labelled by colours $\{0,1,2, \ldots, n\}$ so that every $n$-simplex has a vertex of each colour. If $\bar{K}$ contains exactly $n+1$ vertices, then it is said to be contracted. Note that every $n$-manifold $M$ admits coloured triangulations: it is sufficient to consider the first barycentric subdivision $K^{\prime}$ of any triangulation $K$ of $M$, and to label every vertex $v$ of $K^{\prime}$ with the dimension of the simplex $\sigma$ of $K$ whose barycenter is $v$.

Surprisingly enough, the results related to the average edge order of coloured triangulations of 3-manifolds are analogous to Luo-Stong' ones:

THEOREM II. Let $\bar{K}$ be any coloured triangulation of a closed connected 3-manifold M. Then:

(a) $2 \leq \mu_{0}(\bar{K})<6$, equality holds if and only if $\bar{K}$ is the coloured triangulation of $\mathbb{S}^{3}$ consisting of two 3-simplices with identified boundaries.

(b) For any $\epsilon>0$, there are coloured triangulations $\bar{K}_{1}$ and $\bar{K}_{2}$ of $M$ such that $\mu_{0}\left(\bar{K}_{1}\right)<4+\epsilon$ and $\mu_{0}\left(\bar{K}_{2}\right)>6-\epsilon$.

(c) If $\mu_{0}(\bar{K})<4$, then $\bar{K}$ is a coloured triangulation of $\mathbb{S}^{3}$.

(d) If $\mu_{0}(\bar{K})=4$, then $\bar{K}$ is a coloured triangulation of $\mathbb{S}^{3}, \mathbb{S}^{2} \times \mathbb{S}^{1}, \mathbb{S}^{2} \tilde{\times} \mathbb{S}^{1}$, or $\mathbb{R} \mathbb{P}^{3}$ (where $\mathbb{R P}^{3}$ denotes the 3-dimensional real projective space).

Furthermore, in the last three cases, the induced contracted triangulations are well determined.

The above theorem may be proved with little effort by means of the representation theory of PL-manifolds via edge-coloured graphs (see, for details, [3], [1], [7], [12] and their bibliography).

If $\bar{K}$ is a given coloured triangulation of an $n$-manifold $M$, then the 1-skeleton of the dual of $\bar{K}$ is a multigraph (i.e. without loops, but possibly with multiple edges) regular of degree $n+1$, whose edges may be properly coloured by the following rule: if $e$ is the edge dual to an $(n-1)$-face $A^{n-1}$ of $\bar{K}$, then $e$ inherits the colour of each vertex $v$ of $\bar{K}$ such that $v A^{n-1}$ is an $n$-simplex of $\bar{K}$. The resulting edge-coloured graph $\Gamma=\Gamma(\bar{K})$ is said to be an $(n+1)$-coloured graph representing $M$ (or an $n$-gem of $M$ ), since the above construction can be easily reversed to reobtain the coloured triangulation $\bar{K}=K(\Gamma)$ of the $n$-manifold $M$. Moreover, $\Gamma$ is a bipartite graph if and only if the represented manifold is orientable.

If $(\Gamma, \gamma)$ is an $(n+1)$-coloured graph, and $\mathcal{B} \subset \Delta_{n}$ is a subset of the colour set with cardinality $\# \mathcal{B}=m \leq n$, then $\Gamma_{\mathcal{B}}$ will denote the $m$-coloured graph $\left(V(\Gamma), \gamma^{-1}(\mathcal{B})\right)$; each connected component of $\Gamma_{\mathcal{B}}$ will be called a $\mathcal{B}$-residue, or an m-residue. An h-dipole $(1 \leq h \leq n)$ of $(\Gamma, \gamma)$ is a subgraph $\Theta$ of $\Gamma$ consisting of two vertices $x, y$ joined by $h$ edges with colours $c_{1}, c_{2}, \ldots, c_{h} \in \Delta_{n}$, such that $x, y$ belong to distinct $\left(\Delta_{n}-\left\{c_{1}, \ldots, c_{h}\right\}\right)$ residues. The elimination of an $h$-dipole $\Theta$ consists of deleting the subgraph $\Theta$ from $\Gamma$ 
and welding the "hanging" edges of the same colour. The inverse process is said to be the addition of an $h$-dipole $\Theta$. In [2], $h$-dipole eliminations and additions $(1 \leq h \leq n)$ are proved to be the elementary moves connecting $n$-gems of the same $n$-manifold.

\section{Proof of Theorem II.}

ProOF OF (a). Let $\Gamma=\Gamma(\bar{K})$ be the 3-gem associated to the given coloured triangulation of $M$, and let $p=\# V(\Gamma)$ (resp. $g_{i j}, i, j \in \Delta_{3}=\{0,1,2,3\}$ ) denote the order of $\Gamma$ (resp. the number of $\{i, j\}$-residues of $\Gamma$ ). By construction, it is easy to check that the number of edges of $\bar{K}$ equals the total number of 2-residues of $\Gamma$, while the number of triangles of $\bar{K}$ equals $2 p$ (i.e. the number of edges of $\Gamma$ ).

Thus, $\mu_{0}(\bar{K})=\frac{3 F_{0}(\bar{K})}{E_{0}(\bar{K})}=\frac{3(2 p)}{\sum_{i, j} g_{i j}}$.

In [4], the following relation is proved to characterize 3-gems among 4-coloured graphs:

$$
\sum_{i, j} g_{i j}=\sum_{i} g_{\hat{i}}+p
$$

where $g_{\hat{i}}\left(i \in \Delta_{3}\right)$ denotes the number of $\left(\Delta_{3}-\{i\}\right)$-residues of $\Gamma$.

This yields:

$$
\mu_{0}(\bar{K})=\frac{6 p}{\sum_{i} g_{\hat{i}}+p}
$$

Hence, $2=\frac{6 p}{2 p+p} \leq \mu_{0}(\bar{K})<\frac{6 p}{p}=6$ directly follows from $0<\sum_{i} g_{\hat{i}} \leq 2 p$.

In particular, $\mu_{0}(\bar{K})=2$ means $\sum_{i} g_{\hat{i}}=2 p$, i.e. every 3-residue of $\Gamma$ has order two. It is easy to check that this happens if and only if $\Gamma$ is the order two 4-coloured graph depicted in Figure 1, which is obviously associated to the coloured triangulation of $\mathbb{S}^{3}$ consisting of two 3-simplices with identified boundaries.
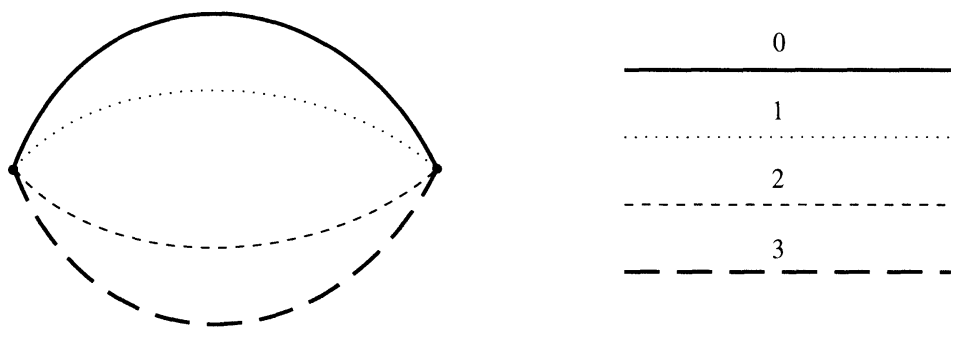

FIGURE 1

PROOF OF (b). Let $\bar{K}$ be a coloured triangulation of $M$, and let $e$ be an edge of $\bar{K}$, with end-points $v_{i}, v_{j}$, labelled by colours $i, j \in \Delta_{3}$ respectively. The coloured trisection of the edge $e$ is the process consisting of a stellar subdivision on $e$-which has exactly 
one new $i$-labelled vertex $w_{i}$,--followed by a new one on the edge $e^{\prime}$ with end-points $v_{i}, w_{i}$-which has exactly one new $j$-labelled vertex $w_{j}$.

The construction of the associated $n$-gem $\bar{\Gamma}=\Gamma(\bar{K})$ allows to verify that the edge $e$ is represented in $\bar{\Gamma}$ by an $\left(\Delta_{3}-\{i, j\}\right)$-residue $R$, whose length equals the order of $e$ in $\bar{K}$ (i.e. the number of triangles of $\bar{K}$ containing it). Figure 2(a) (resp. 2(b)) shows the local effect of the described trisection on a single 3-simplex of $\bar{K}$ containing the edge $e$ (resp. on a single vertex of the residue $R$ of $\bar{\Gamma}$ ), while Figure 2(c) shows the global effect of the same trisection on the whole residue $R$ of $\bar{\Gamma}$, assuming the length of $R$ to be six: see [5] for more details.

It is now easy to check that, if $a \geq 2$ is the order of the edge $e$ in $\bar{K}$, then the coloured triangulation $\bar{K}^{n}$ of $M$ obtained from $\bar{K}$ by performing $n$ coloured trisections on $e$, has

$$
\mu_{0}\left(\bar{K}^{n}\right)=\frac{3\left(F_{0}(\bar{K})+4 a n\right)}{E_{0}(\bar{K})+(2 a+2) n}
$$

since each operation increases $g_{\hat{i}}$ and $g_{\hat{j}}$ by one and $p$ by $2 a$.

In case $a=2$, we have $\lim _{n \rightarrow \infty} \mu_{0}\left(\bar{K}^{n}\right)=4$; this ensures the existence of $\bar{K}_{1}$ with $\mu_{0}\left(\bar{K}_{1}\right)<4+\epsilon$, since any 3-manifold $M$ admits a coloured triangulation with an order two edge (it is sufficient to add a 2-dipole to any 3-gem of $M$ ).

On the other hand, the existence of $\bar{K}_{2}$ with $\mu_{0}\left(\bar{K}_{2}\right)>6-\epsilon$ follows from

$$
\lim _{a \rightarrow \infty}\left(\lim _{n \rightarrow \infty} \mu_{0}\left(\bar{K}^{n}\right)\right)=6
$$

and from the fact that every 3-manifold $M$ admits a coloured triangulation with an edge of arbitrarily high order (it is sufficient to add a suitable sequence of 3-dipoles to any 3-gem of $M$ ).

PrOOF OF (c) AND (d). Let $\bar{K}$ be a coloured triangulation of $M$, and let $\bar{K}^{(n)}$ be the coloured triangulation of $M$ obtained from $\bar{K}$ by performing $n$ 1-dipole eliminations; since the elimination of an $i$-coloured 1-dipole decreases $g_{\hat{i}}$ by one and $p$ by two, then:

$$
\mu_{0}\left(\bar{K}^{(n)}\right)=\frac{3\left(F_{0}(\bar{K})-4 n\right)}{E_{0}(\bar{K})-3 n} .
$$

If $\mu_{0}(\bar{K})<4$ (resp. $\mu_{0}(\bar{K})=4$ ) is assumed, $\mu_{0}\left(\bar{K}^{(n)}\right)<4\left(\right.$ resp. $\left.\mu_{0}\left(\bar{K}^{(n)}\right)=4\right)$ immediately follows, for every integer $n$ satisfying the trivial condition $0 \leq 3 n<E_{0}(\bar{K})$.

Thus, for every $i \in \Delta_{3}$, the number $g_{\hat{i}}$ may be reduced by means of 1-dipole eliminations, till a 3-gem $\tilde{\Gamma}$ of $M$ is obtained, with $\mu_{0}(K(\tilde{\Gamma}))<4\left(\operatorname{resp} . \mu_{0}(K(\tilde{\Gamma}))=4\right)$ and $g_{\hat{i}}=1, \forall i \in \Delta_{3}$. If $\tilde{p}$ denotes the order of $\tilde{\Gamma}$, then formula (\#) gives

$$
\mu_{0}(K(\tilde{\Gamma}))=\frac{6 \tilde{p}}{4+\tilde{p}}
$$


and $\tilde{p}<8$ (resp. $\tilde{p}=8$ ) follows. Hence, in order to establish the thesis, we have to study all the possible 3 -gems $\tilde{\Gamma}$ with $g_{\hat{i}}=1, \forall i \in \Delta_{3}$ (i.e. all the possible 3-manifold crystallizations: see [10]) up to order eight.

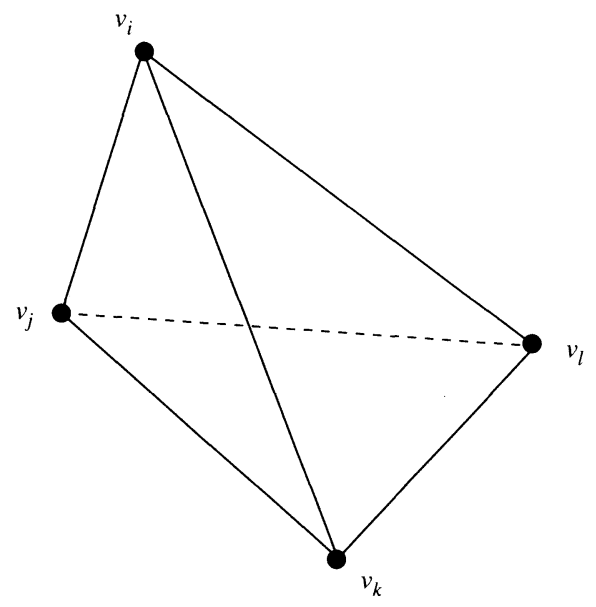

FIGURE 2(a)

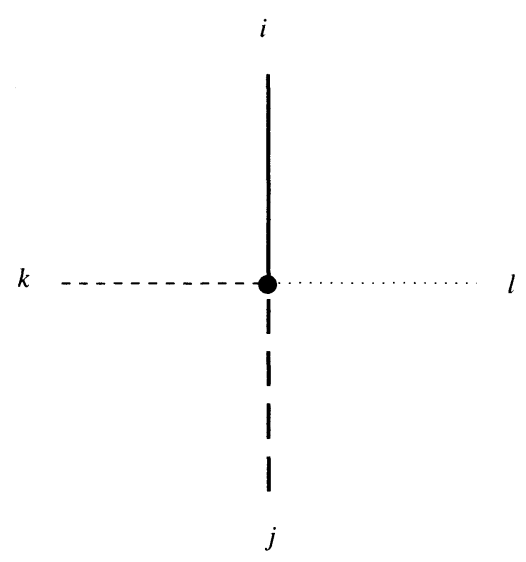

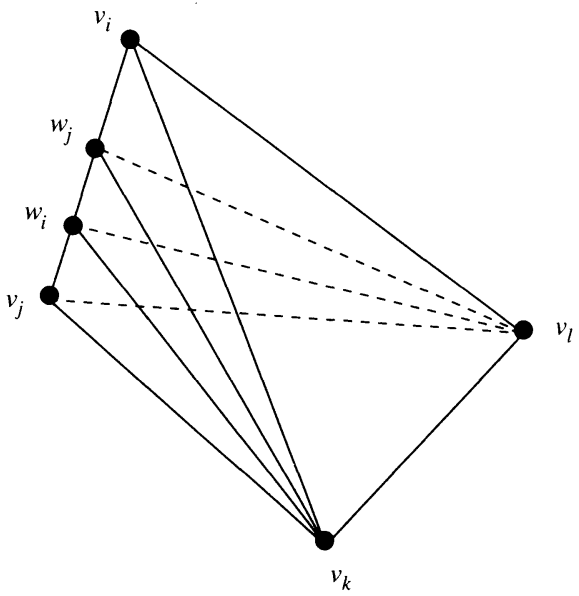

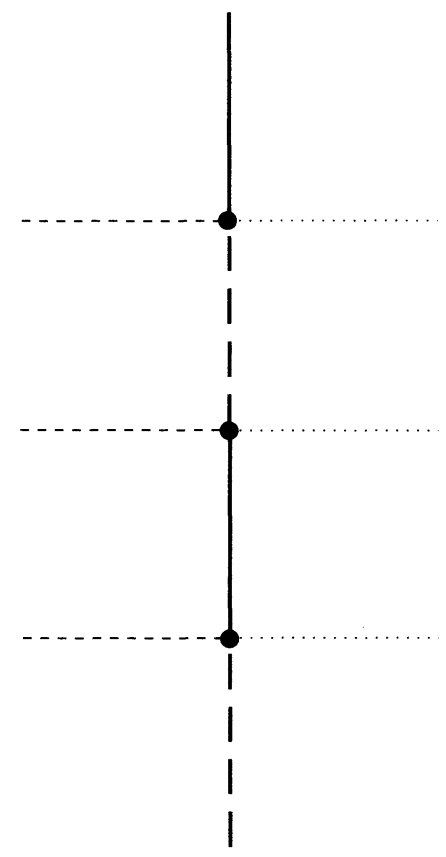

FIGURE 2(b) 


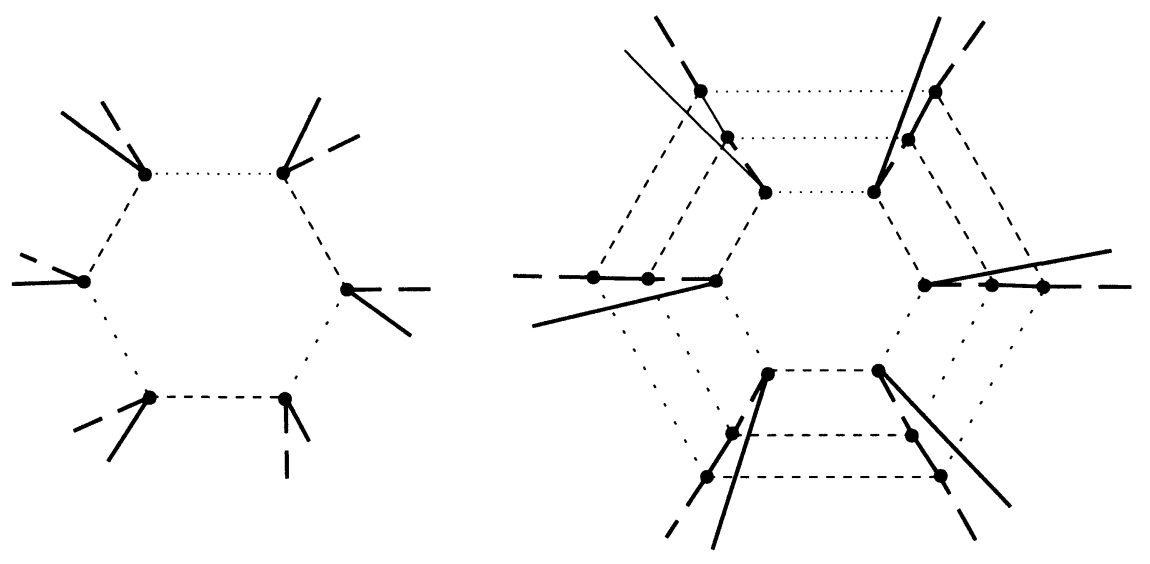

FIGURE 2(c)

The case $\tilde{p}=2$ (i.e. $\mu_{0}(K(\tilde{\Gamma}))=2$ ), has already been considered, and directly produces $M \cong \mathbb{S}^{3}$.

As far as $\tilde{p} \geq 4$ is concerned, it is necessary to remember that each 3-manifold crystallization satisfies the following properties, for every permutation $\epsilon=\left(\epsilon_{0}, \epsilon_{1}, \epsilon_{2}, \epsilon_{3}\right)$ of $\Delta_{3}$ (see [4]):

(*) $g_{\epsilon_{0} \epsilon_{1}}=g_{\epsilon_{2} \epsilon_{3}}$;

(**) $g_{\epsilon_{0} \epsilon_{1}}+g_{\epsilon_{0} \epsilon_{2}}+g_{\epsilon_{0} \epsilon_{3}}=2+\tilde{p} / 2$.

If $\tilde{p}=4$ (resp. $\tilde{p}=6$ ) is assumed, then property (**) implies the existence of two colours $i, j \in \Delta_{3}$ such that $g_{i j}=2$ (resp. $g_{i j} \geq 2$ ); this obviously implies that any (resp. at least one) $\{i, j\}$-residue of $\tilde{\Gamma}, R$ say, has length two. On the other hand, property (*) gives $g_{\Delta_{3}-\{i, j\}}=g_{i j}=2$ (resp. $g_{\Delta_{3}-\{i, j\}}=g_{i j} \geq 2$ ), and hence each $\left(\Delta_{3}-\{i, j\}\right)$-residue of $\tilde{\Gamma}$ has length $\leq 4$. This ensures that the two multiple edges of $R$ constitute a dipole: in fact, if the vertices of $R$ would belong to the same length four $\left(\Delta_{3}-\{i, j\}\right)$-residue, then the (unique) $\left(\Delta_{3}-\{i\}\right)$-residue $\tilde{\Gamma}_{\hat{i}}$ of $\tilde{\Gamma}$ could not be a bipartite graph (against the hypothesis that $\tilde{\Gamma}$ is a 3-manifold crystallization, which implies $K\left(\tilde{\Gamma}_{\hat{i}}\right) \cong S^{2}$ : see [3] for details). Then, since the elimination of the 2-dipole $R$ decreases the order of $\tilde{\Gamma}, M \cong \mathbb{S}^{3}$ follows by iteration; this completes the proof of (c).

Let us now assume $\tilde{p}=8$. If there exist two colours $i, j \in \Delta_{3}$ such that $g_{i j} \geq 3$, then $\tilde{\Gamma}$ results to contain two multiple edges (of colours $i, j$ ) constituting a 2-dipole (by properties $(*)$ and $(* *)$, via considerations perfectly analogous to the previous ones); this yields $M \cong \mathbb{S}^{3}$. On the other hand, if there exist two colours $i, j \in \Delta_{3}$ such that $g_{i j}=1$, then property (**) implies the existence of $k \in \Delta_{3}-\{i, j\}$ such that $g_{i k} \geq 3$; so, $M \cong \mathbb{S}^{3}$ follows, too. The last case to be analyzed, i.e. $g_{i j}=2, \forall i, j \in \Delta_{3}$, admits the following subcases:

1) $\tilde{\Gamma}$ contains 2 -dipoles (and this again implies $M \cong \mathbb{S}^{3}$ ); 
2) every 2-residue of $\tilde{\Gamma}$ has length four (and this occurs only in the crystallization of $\mathbb{R} \mathbb{P}^{3}$ depicted in Figure 3);

3) $\tilde{\Gamma}$ lacks 2-dipoles, but it contains at least a pair of multiple edges.

It is now easy to check that the third situation implies the existence of a permutation $\epsilon=\left(\epsilon_{0}, \epsilon_{1}, \epsilon_{2}, \epsilon_{3}\right)$ of $\Delta_{3}$ such that, for every $i \in \Delta_{3}$, one $\left\{\epsilon_{i}, \epsilon_{i+1}\right\}$-residue has length two and the other one has length six, while every $\left\{\epsilon_{i}, \epsilon_{i+2}\right\}$-residue has length four; thus, the only possible crystallizations are depicted in Figure 4 and Figure 5, and respectively represent $\mathbb{S}^{2} \times \mathbb{S}^{1}$ and $\mathbb{S}^{2} \tilde{\times} \mathbb{S}^{1}$.

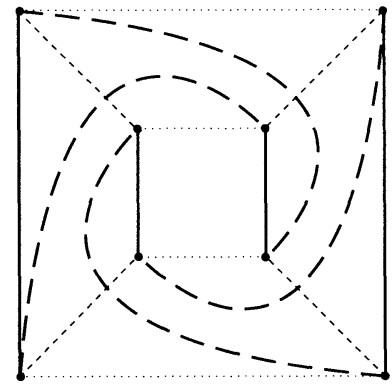

FIGURE 3

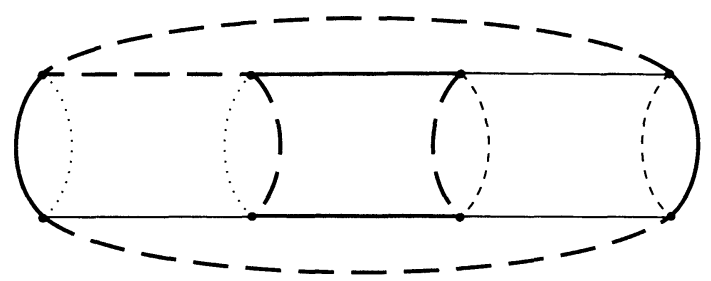

FIGURE 4

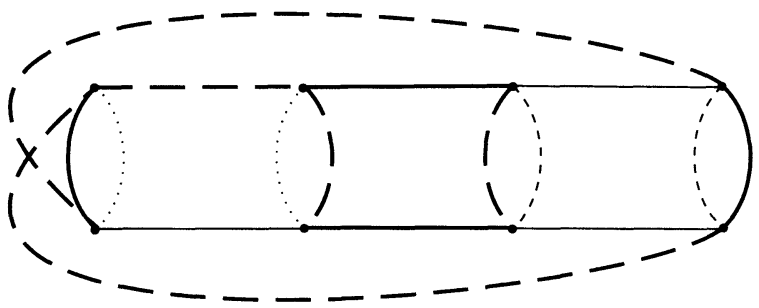

FIGURE 5

Hence, apart from the case $M \cong \mathbb{S}^{3}$, the starting coloured triangulation $\bar{K}$ of $M \cong \mathbb{R} \mathbb{P}^{3}$ $\left(\right.$ resp. $\left.M \cong \mathbb{S}^{2} \times \mathbb{S}^{1}\right)\left(\right.$ resp. $\left.M \cong \mathbb{S}^{2} \tilde{\times} \mathbb{S}^{1}\right)$ induces the coloured triangulation $\tilde{K}=K(\tilde{\Gamma})$ associated to the graph of Figure 3 (resp. 4) (resp. 5), which is contracted (since it contains 
exactly four vertices, as every triangulation associated to a crystallization: see [10]) and is well-determined by the graph itself.

The theorem is now completely proved.

\section{REFERENCES}

1. J. Bracho and L. Montejano, The combinatorics of colored triangulations of manifolds, Geom. Dedicata 22(1987), 303-328.

2. M. Ferri and C. Gagliardi, Crystallization moves, Pacific J. Math. 100(1982), 233-246.

3. M. Ferri, C. Gagliardi and L. Grasselli, A graph-theoretical representation of PL-manifolds. A survey on crystallizations, Aequationes Math. 31(1986), 121-141.

4. C. Gagliardi, Regular imbeddings of edge-coloured graphs, Geom. Dedicata 11(1981), 397-414.

5. On a class of 3-dimensional polyhedra, Ann. Univ. Ferrara Sez. VII 33(1987), 51-88.

6. P. J. Hilton and S. Wylie, An introduction to algebraic topology-Homologytheory, Cambridge Univ. Press, 1960.

7. S. Lins and A. Mandel, Graph-encoded 3-manifolds, Discrete Math. 57(1985), 261-284.

8. F. Luo and R. Stong, Combinatorics of triangulations of 3-manifolds, Trans. Amer. Math. Soc. 337(1993), 891-906.

9. T. Oda, Convex bodies and algebraic geometry, Springer-Verlag, 1985.

10. M. Pezzana, Diagrammi di Heegaard e triangolazione contratta, Boll. Un. Mat. Ital. 12(1975), 93-105.

11. C. Rourke and B. Sanderson, Introduction to Piecewise-linear Topology, Springer-Verlag, 1972.

12. A. Vince, Combinatorial maps, J. Combin. Theory Ser. B 34(1983), 1-21.

Dipartimento di Matematica Pura ed Applicata

Università di Modena

Via Campi $213 B$

I-41100 Modena

Italy 